\title{
Cardiopatia congênita: estado nutricional e proporcionalidade corporal ao nascimento
}

\author{
Congenital heart disease: nutritional status and body proportionality at birth
}

DOI: $10.37111 /$ braspenj.2020351004

Izabela dos Santos Pereira

Cláudia Porto Sabino Pinho²

Adriana César da Silveira ${ }^{3}$

Unitermos:

Estado nutricional. Peso ao nascer. Peso fetal.

\section{Keywords:}

Nutritional status. Birth weight. Fetal weight.

\section{Endereço para correspondência:}

Cláudia Porto Sabino Pinho

Rua dos Palmares, $\mathrm{s} / \mathrm{n}^{\circ}$ - Santo Amaro - Recife, $\mathrm{PE}$, Brasil - CEP: 50100-060.

E-mail: claudiasabinopinho@hotmail.com

\section{Submissão}

18 de novembro de 2019

Aceito para publicação

17 de março de 2020

\section{RESUMO}

Introdução: As cardiopatias congênitas têm grande impacto nas taxas de mortalidade perinatal e infantil no Brasil. São doenças que podem levar ao comprometimento do crescimento e do desenvolvimento da criança. $O$ objetivo deste estudo é descrever a condição nutricional e a proporcionalidade corporal ao nascimento de crianças portadoras de cardiopatia congênita. Método: Estudo transversal, retrospectivo, utilizando dados ao nascimento de crianças com cardiopatia congênita admitidos num Serviço de Cardiologia no Nordeste brasileiro entre 2011 e 2014. O estado nutricional foi avaliado pelos indicadores peso/idade (P/I), comprimento/idade, peso/comprimento, índice de massa corpórea/idade (IMC/I), índice ponderal, e perímetro cefálico para idade. Resultados: Foram avaliados 117 pacientes, sendo $60,7 \%$ do sexo masculino e $20,9 \%$ com cardiopatia cianótica. Verificou-se que $6,8 \%$ das crianças apresentaram baixo peso ao nascer. Os índices nutricionais demonstraram 3,4\% de déficit no indicador $\mathrm{P} / \mathrm{l} ; 5,1 \%$ no peso/ comprimento; $4,3 \%$ no IMC/I; 7,8\% no comprimento/idade. $O$ índice ponderal demonstrou que $28,2 \%$ das crianças foram desproporcionais ao nascimento e $84,4 \%$ tiveram o perímetro cefálico normal. Menores valores de escore $Z$ dos indicadores $P / I(p=0,030)$ e IMC/I $(p=0,023)$ para as cardiopatias cianóticas, quando comparadas aos outros tipos de cardiopatias congênitas foram observados em nosso estudo. Conclusões: Crianças com cardiopatias congênitas apresentaram condição nutricional ao nascimento relativamente preservada, com prevalência de distúrbios nutricionais semelhante às descritas na literatura.

\section{ABSTRACT}

Introduction: Congenital heart diseases have a great impact on perinatal and infant mortality rates in Brazil. These are diseases that can lead to compromised growth and development of the child. The purpose of this study is to describe the nutritional condition and the corporal proportionality to the birth of children with congenital heart disease. Methods: A cross-sectional, retrospective study using data on the birth of children with congenital heart disease admitted to a cardiology service in the Northeast of Brazil between 2011 and 2014. The nutritional status was assessed by weight/age (W/A) indicators, length/age, weight/length, body mass index/age (BMI/A), ponderal index, cephalic perimeter. Results: A total of 117 patients were evaluated, being $60.7 \%$ male and $20.9 \%$ with cyanotic heart disease. It was found that $6.8 \%$ of the children were low birth weight. The nutritional indexes showed a $3.4 \%$ deficit in the W/A indicator, in weight/length $5.1 \%$, for $\mathrm{BMI} / \mathrm{A} 4.3 \%$ and length/age $7.8 \%$. The ponderal index showed that $28.2 \%$ of the children were disproportionate at birth and $84.4 \%$ had a normal head circumference. Lower $Z$ score values of W/A $(p=0.030)$ and BMI/A $(p=0.023)$ for cyanotic heart disease compared to other types of congenital heart diseases were observed in our study. Conclusions: Children with congenital heart disease had relatively preserved nutritional status at birth, with prevalence of nutritional disorders similar to those described in the literature.

1. Nutricionista Residente do Programa de Residência em Nutrição do Pronto Socorro Cardiológico Universitário de Pernambuco, Universidade de Pernambuco, Recife, PE, Brasil.

2. Doutora em Nutrição pela Universidade Federal de Pernambuco, Nutricionista do Pronto Socorro Cardiológico Universitário de Pernambuco, Universidade de Pernambuco, Recife, PE, Brasil.

3. Doutora em Nutrição pela Universidade Federal de Pernambuco, Nutricionista do Hospital Universitário Walter Cantídio, Universidade Federal do Ceará, Fortaleza, CE, Brasil. 


\section{INTRODUÇÃO}

A nível mundial, a cada 130 milhões de nascidos vivos, cerca de 4 milhões (7\%) de óbitos ainda no período neonatal são relacionados às cardiopatias congênitas. Com o desenvolvimento da tecnologia em saúde, essas doenças podem ser diagnosticadas no período fetal'. Define-se cardiopatia congênita como uma malformação estrutural do coração ou dos grandes vasos intratorácicos, que podem cursar com alterações funcionais ${ }^{2}$. São classificadas em dois grandes grupos: cianóticas e acianóticas, onde a primeira é caracterizada por levar a uma maior repercussão hemodinâmica. Algumas cardiopatias congênitas evolvem com insuficiência cardíaca ainda na vida fetal, como a estenose aórtica crítica ${ }^{3}$.

Um fator agravante é o gasto energético aumentado em torno de $40 \%$, principalmente nas cardiopatias cianóticas e naqueles que apresentam insuficiência cardíaca e hipertensão pulmonar, condições associadas a maior trabalho que 0 coração exerce para realizar suas funções ${ }^{4}$. $\bigcirc$ grau de comprometimento do crescimento e desenvolvimento depende do número de defeitos cardíacos, sendo maior naquelas com malformações múltiplas ${ }^{4}$. Além disso, a ingestão alimentar deficiente, que pode ser frequentemente observada nessa população, também contribui para o déficit nutricional ${ }^{3,5}$.

A prevalência da desnutrição em portadores de cardiopatias congênitas em países em desenvolvimento é elevada, variando entre $27 \%$ e $85 \% 6$ e, no Brasil, de $46,6 \%$ a $83,3 \% 5$. Evidências sugerem que pode haver comprometimento do estado nutricional já na vida intrauterina.

Para a avaliação do estado nutricional ao nascimento, índices de proporcionalidade corporal podem ser utilizados, como o índice ponderal (IP) ou índice de Rohrer e a razão peso/comprimento ${ }^{7}$. O IP é definido como o peso ao nascimento em gramas vezes cem, dividido pelo comprimento ao nascer ao cubo (em centímetros), e é utilizado para relacionar diferentes dimensões do crescimento fetal, principalmente o retardo de crescimento intrauterino, determinar o tipo de desnutrição sofrida na vida intrauterina, e a sua relação com a mortalidade neonatal ${ }^{8}$.

Devido ao grande impacto nas taxas de mortalidade perinatal e infantil no Brasil, conhecer as características dos portadores de cardiopatia congênita ao nascimento pode auxiliar em seus cuidados imediatos, sobretudo naqueles que precisam ser submetidos a cirurgias nos primeiros meses de vida, para a prevenção precoce do comprometimento nutricional, representando melhoria da qualidade de vida destes indivíduos ${ }^{9,10}$. Nesse contexto, o objetivo desse estudo é descrever a condição nutricional e a proporcionalidade corporal ao nascimento de crianças portadoras de cardiopatia congênita.

\section{MÉTODO}

Foi realizado um estudo descritivo, transversal, com análise retrospectiva de dados de pacientes atendidos na Unidade de Cardiologia Pediátrica de um hospital referência em Cardiologia no Nordeste brasileiro. Os dados do nascimento foram coletados retrospectivamente do prontuário ou caderneta da criança admitida na instituição para tratamento cirúrgico, no período de maio de 2011 a outubro de 2014.

O tamanho amostral mínimo foi estimado tendo como base a prevalência de baixo peso ao nascer em crianças com cardiopatias congênitas. Desse modo, com base nas fórmulas de Lwanga \& Tye, adotando-se uma prevalência de $8 \%{ }^{11} \mathrm{com}$ uma margem de erro aceitável de $5 \%$, uma confiabilidade de 95\%, o tamanho amostral mínimo foi estimado em 113 pacientes. Para cobrir eventuais perdas, esse número foi aumentado em $10 \%$, totalizando um n de 125 pacientes.

Foram excluídos pacientes nascidos de gestações múltiplas, pré-termo, portadores de outras malformações congênitas que levem ao retardo do crescimento intrauterino (RCIU), bem como portadores de síndromes genéticas ou aquelas com ausência de informação de algumas das variáveis do nascimento.

Com as medidas antropométricas do nascimento (peso e comprimento) determinou-se o estado nutricional, a partir do escore $Z$, utilizando-se os índices peso por idade $(\mathrm{P} / \mathrm{I})$, comprimento por idade $(C / I)$, peso por comprimento $(P / C)$, e o índice de massa corporal (IMC) para idade (IMC/I). Foram considerados os pontos de corte para categorização dos resultados, sendo definido déficit de nutricional quando o escore $\mathrm{z}<-2$, adequado os valores de escore $\mathrm{z}$ entre -2 e $<2$ escores e excesso de peso quando escore $z \geq 2$, estabelecidos para crianças, tendo como padrão de referência as Curvas de Crescimento da Organização Mundial da Saúde ${ }^{12}$, por meio do software Anthrov3.2.2.

A classificação do recém-nascido segundo o peso ao nascer foi realizada considerando-se: o baixo peso as crianças com menos de $2500 \mathrm{~g}$ e não baixo peso aquelas nascidas com peso igual ou superior a $2500 \mathrm{~g}$. Para o cálculo da proporcionalidade corporal foram utilizados $\circ \mathrm{IP}^{7}$ e a razão peso/comprimento. O IP foi calculado através da fórmula: $\mathrm{IP}=\mathrm{PN}(\mathrm{g}) \times 100 / \mathrm{CN}^{3}(\mathrm{~cm})$. Onde: $\mathrm{PN}=$ peso ao nascer, $\mathrm{CN}$ = comprimento ao nascer, sendo classificadas como desproporcionais as crianças com IP $<2,49$, que são aquelas com baixo peso e comprimento normal, ou proporcionais, quando o $\mathbb{P} \geq 2,49^{13}$, estas são aquelas com baixo peso e baixo comprimento ao nascer. $O$ ponto de corte razão peso/comprimento foi definido pelo menor tercil para a população total $(<62,6)$.

A classificação do perímetro cefálico (PC) foi realizada de acordo com o ponto de corte de $32 \mathrm{~cm}$, para ambos os 
sexos. A avaliação do PC para idade (PC/I) considerou a classificação: pequena para idade $<-2$ escores $\mathrm{z}$; adequado: de -2 escores $z$ a $<2$ escores; grande para idade: $>2$ escores $z$, estabelecidos para crianças $^{14}$.

Em relação à idade gestacional, foram classificados como prematuros, os pacientes nascidos com menos de 37 semanas, segundo o Ministério da Saúde. As outras variáveis consideradas foram: os antecedentes perinatais: paridade (1 filho = nulípara, 2 ou $+=$ multípara), tipo de parto (natural ou cirúrgico), uso ou não pela mãe de drogas lícitas (álcool e tabaco), ilícitas (maconha, crack, cola de sapateiro, heroína, cocaína) ou medicamentos durante a gestação, doenças durante a gestação, e o tipo de cardiopatia (acianótica, cianótica ou mista). $O$ diagnóstico de cardiopatia congênita foi confirmado pelo ecocardiograma realizado pela equipe de pediatria do hospital.

A análise estatística foi realizada no programa estatístico SPSS versão 13.0 (SPSS Inc., Chicago, IL, USA). As variáveis contínuas foram testadas quanto à normalidade de distribuição pelo teste de KolmogorovSmirnov e quando apresentaram distribuição normal foram descritas na forma de média e desvio padrão. Para comparação das médias foi empregado o teste " $t$ " de Student (duas médias) e ANOVA (duas ou mais médias). Quando os critérios paramétricos (distribuição normal e homocedasticidade) não foram atingidos, os testes não paramétricos de Mann Whitney e Kruskal-Wallis foram utilizados. O teste do Qui-Quadrado $\left(\chi^{2}\right)$ ou Exato de Fisher foi empregado para comparação de frequências. Foi adotado o nível de significância de 5\% para rejeição da hipótese de nulidade.

A pesquisa foi aprovada pelo Comitê de Ética em Pesquisa do Complexo Hospitalar do HUOC/Procape, conforme Resolução 466/12, sob protocolo 856.727 de novembro de 2014 (CAAE:277221 14.3.0000.5192).

\section{RESULTADOS}

No período do estudo, 308 pacientes com cardiopatia congênita foram admitidos na Unidade de Cardiologia Pediátrica do hospital, entretanto, 193 tiveram que ser excluídos por síndromes genéticas $(n=47)$, prematuridade $(n=18)$, gemelaridade $(n=5)$, e a maioria $(n=113)$ por falta dos dados antropométricos ao nascimento, seja por falta de registro ou por não estarem com suas cadernetas de saúde. Foram incluídos 125 pacientes e, depois de eliminadas as perdas e inconsistência de informações, o tamanho amostral foi de 117 pacientes.

Deste total, a maioria era do sexo masculino $(60,7 \%)$, procedente do estado de Pernambuco $(70,7 \%)$, de mães multíparas $(62,7 \%)$ e que realizaram o pré-natal $(96,3 \%)$ (Tabela 1).
Tabela 1 - Caracterização demográfica e dos antecedentes perinatais de crianças com cardiopatia congênita admitidas em um centro de referência no Nordeste brasileiro, entre maio de 2011 e outubro de 2014.

\begin{tabular}{lcc}
\hline Variável & $\mathbf{n}$ & $\%$ \\
\hline Sexo & 71 & 60,7 \\
Masculino & 46 & 39,3 \\
$\quad$ Feminino & 82 & 70,7 \\
\hline Origem & 34 & 29,3 \\
$\quad$ Pernambuco & \\
Outros Estados & 103 & 96,3 \\
\hline Pré-Natal & 4 & 3,7 \\
Sim & & \\
Não & 61 & 56,6 \\
\hline Parto & 47 & 43,5 \\
Normal & & \\
Cesáreo & 41 & 37,3 \\
Paridade & 69 & 62,7 \\
Nulípara & \\
$\quad$ Multípara & 13 & 11,9 \\
\hline Uso de droga ilícita na gestação & 88,1 \\
Sim & 96 & \\
Não & &
\end{tabular}

Em relação à cardiopatia congênita, foi identificado que $20,9 \%$ apresentavam cardiopatia cianótica e 36,5\% tinham cardiopatia complexa. As cardiopatias mais prevalentes foram a tetralogia de Fallot (12,8\%), seguida das estenoses aórtica e pulmonar não críticas (Tabela 2).

Tabela 2 - Frequência das cardiopatias observadas na amostra de crianças com cardiopatia congênita admitidas em um centro de referência no Nordeste brasileiro, entre maio de 2011 e outubro de 2014.

\begin{tabular}{lcc}
\hline Malformação cardíaca & $\mathbf{n}$ & $\%$ \\
\hline Tetralogia de Fallot & 15 & 12,8 \\
Estenoses aórtica e pulmonar não críticas & 13 & 11,1 \\
Comunicação interventricular (CIV) & 12 & 10,2 \\
Comunicação interatrial (CIA) & 11 & 9,4 \\
Transposição das grandes artérias & 9 & 7,7 \\
Atresia tricúspide & 9 & 7,7 \\
Persistência do canal arterial & 7 & 5,9 \\
DVSVD & 7 & 5,9 \\
Atresia pulmonar & 6 & 5,1 \\
CIA+CIV & 6 & 5,1 \\
Desvio de septo atrioventricular (DSAV) & 6 & 5,1 \\
Coarctação de aorta & 6 & 5,1 \\
Drenagem anômala total das veias pulmo- & 3 & 2,5 \\
nares & & \\
Origem anômala da artéria coronária & 1 & 0,85 \\
esquerda & & \\
Insuficiência mitral congênita & 1 & 0,85 \\
Ventrículo esquerdo não compactado & 1 & 0,85 \\
Doença de Ebstein & 1 & 0,85 \\
DSAV + HVE+ EP+ isomerismo esquerdo & 1 & 0,85 \\
Isomerismo direito & 1 & 0,85 \\
Total & 117 & 100 \\
\hline DVSVD = dupla via do saída do ventrículo diroto: EP = cstonose pulmonar; HVE = hipoplasia
\end{tabular}

Salda do ventriculo direito: EP = estenose pulmonar: HVE = hipoplasia do ventrículo esquerdo. 
Tabela 3 - Dados nutricionais ao nascimento de crianças com cardiopatia congênita admitidas em um centro de referência no Nordeste brasileiro, entre maio de 2011 e outubro de 2014.

\begin{tabular}{|c|c|c|}
\hline Variável & $\mathrm{n}$ & $\%$ \\
\hline \multicolumn{3}{|l|}{ Peso ao Nascer (g) } \\
\hline$<2500$ & 8 & 6,8 \\
\hline$>2500$ & 109 & $93,2 \%$ \\
\hline \multicolumn{3}{|l|}{$P / I$} \\
\hline Déficit de peso & 4 & 3,4 \\
\hline Peso adequado & 109 & 93,2 \\
\hline Excesso de peso & 4 & 3,4 \\
\hline \multicolumn{3}{|l|}{$\mathrm{P} / \mathrm{C}$} \\
\hline Déficit de peso & 6 & 5,1 \\
\hline Peso adequado & 106 & 90,6 \\
\hline Excesso de peso & 5 & 4,3 \\
\hline \multicolumn{3}{|l|}{$\mathrm{C} / \mathrm{I}$} \\
\hline Déficit de comprimento & 9 & 7,8 \\
\hline Comprimento adequado & 107 & 92,2 \\
\hline \multicolumn{3}{|l|}{ IMC/I } \\
\hline Déficit de peso & 5 & 4,3 \\
\hline Peso adequado & 107 & 91,4 \\
\hline Excesso de peso & 5 & 4,3 \\
\hline \multicolumn{3}{|l|}{ IP } \\
\hline$<2,49$ & 33 & 28,2 \\
\hline$>2,49$ & 84 & 71,8 \\
\hline \multicolumn{3}{|l|}{ Perímetro cefálico } \\
\hline$\leq 32 \mathrm{~cm}$ & 14 & 15,2 \\
\hline$>32 \mathrm{~cm}$ & 78 & 84,8 \\
\hline \multicolumn{3}{|l|}{ Razão peso/comprimento } \\
\hline Menor tercil & 39 & 33,3 \\
\hline $2^{\circ}$ e $3^{\circ}$ tercil & 78 & 66,7 \\
\hline
\end{tabular}

Verificou-se prevalência de 6,8\% de baixo peso ao nascer $(<2500$ g). Em relação aos índices nutricionais, o que apresentou maior percentual de déficit nutricional $(5,1 \%)$ foi o indicador P/C. Quanto ao índice ponderal, 28,2\% da população estudada foram classificados como desproporcional. A maioria $(84,8 \%)$ das crianças apresentou perímetro cefálico normal. Os dados nutricionais estão representados na Tabela 3.

Quando comparados os dados nutricionais por sexo, não se verificaram diferenças estatisticamente significantes entre os índices antropométricos (Tabela 4). No entanto, quando os dados foram estratificados segundo o tipo de cardiopatia, foi observado que aqueles com cardiopatia cianótica apresentaram menores médias de escore $Z$ dos índices $P / I(p=0,030)$ e IMC/I $(p=0,023)$, quando comparados com pacientes com cardiopatia congênita acianótica e complexa. $\bigcirc$ índice ponderal foi similar nos três grupos de cardiopatias (Tabela 5).

\section{DISCUSSÃO}

O grande número de exclusões $(n=113)$ por falta dos dados antropométricos ao nascimento, seja por falta de registro ou por não estarem com suas cadernetas de saúde, revela o desconhecimento das mães quanto à importância destes dados para a saúde de seus filhos. Esse fato é preocupante, pois são informações de extrema relevância para todas as crianças, sobretudo para aquelas com doenças crônicas, como as cardiopatias congênitas, devido a sua alta demanda energética, e a grande probabilidade de cirurgia em algum momento da vida. Aproximadamente 20\% dos pacientes podem ter resolução espontânea do defeito

Tabela 4 - Dados nutricionais ao nascimento estratificado por sexo, de crianças portadoras de cardiopatia congênita admitidas em um centro de referência no Nordeste brasileiro, entre maio de 2011 e outubro de 2014.

\begin{tabular}{lcccc}
\hline Variável & Total & Sexo Masculino & Sexo Feminino & p-valor* \\
\hline Idade (meses) & $29,0(5,0-76,0)$ & $19,0(5,0-66,0)$ & $42,0(6,0-84,0)$ & $0,093^{2}$ \\
PN (g) & $3246,0 \pm 502,4$ & $3268,5 \pm 503,9$ & $3211,2 \pm 503,9$ & $0,549^{1}$ \\
CN (cm) & $49(48,0-50,7)$ & $49(48,0-50,5)$ & $49(47,0-51,0)$ & $0,821^{2}$ \\
IMC (kg/m²) & $13,6 \pm 1,8$ & $13,7 \pm 2,0$ & $13,4 \pm 1,5$ & $0,560^{1}$ \\
SC & $0,2(0,2-0,2)$ & $0,2(0,2-0,2)$ & $0,2(0,2-0,2)$ & $0,900^{2}$ \\
Escore Z P/I & $-0,1 \pm 1,1$ & $-0,2 \pm 1,1$ & $0 \pm 1,1$ & $0,565^{1}$ \\
Escore Z P/C & $0,1 \pm 1,3$ & $0,2 \pm 1,2$ & $-0,1 \pm 1,3$ & $0,202^{1}$ \\
Escore Z C/I & $-0,3 \pm 1,2$ & $-0,3 \pm 1,1$ & $-0,2 \pm 1,4$ & $0,509^{1}$ \\
Escore Z IMC/I & $0,1 \pm 1,1$ & $0,1 \pm 1,1$ & $0,1 \pm 1,1$ & $0,887^{1}$ \\
IP & $2,7 \pm 0,4$ & $2,7 \pm 0,4$ & $2,7 \pm 0,3$ & $0,763^{1}$ \\
Escore Z & & $0,2 \pm 1,3$ & $0,173^{1}$ \\
Perímetro & $-0,1 \pm 1,3$ & $-0,2 \pm 1,3$ & & $0,521^{1}$ \\
Cefálico/ Idade & & & $65,6 \pm 8,3$ \\
Razão P/C & $66,3 \pm 8,7$ & $66,7 \pm 9,0$ & \\
\hline CI=compintopar
\end{tabular}


Tabela 5 - Associação de variáveis nutricionais ao nascimento com o tipo de cardiopatia de crianças admitidas em um centro de referência no Nordeste brasileiro, entre maio de 2011 e outubro de 2014.

\begin{tabular}{lcccc}
\hline Variável & Cianótica & Acianótica & Complexa & p-valor* \\
\hline PN (g) & $3062,7 \pm 436,6$ & $3235,3 \pm 487,3$ & $3352,3 \pm 543,5$ & 0,080 \\
CN (cm) & $48,5(48,0-50,0)$ & $49,0(47,0-50,7)$ & $49,5(48,0-51,0)$ & 0,441 \\
IP & $2,7 \pm 0,3$ & $2,7 \pm 0,5$ & $2,8 \pm 0,3$ & 0,682 \\
IMC (kg/m²) & $12,9 \pm 1,4$ & $13,6 \pm 1,4$ & $13,7 \pm 1,5$ & 0,094 \\
Escore Z P/I & $-0,6 \pm 0,9^{\mathrm{a}}$ & $-0,1 \pm 1,0^{\mathrm{b}}$ & $0,1 \pm 1,2^{\mathrm{b}}$ & 0,030 \\
Escore Z P/C & $-0,2 \pm 1,2$ & $0,1 \pm 1,3$ & $0,2 \pm 1,2$ & 0,478 \\
Escore Z C/I & $-0,5 \pm 0,9$ & $-0,3 \pm 1,3$ & $0,2 \pm 1,2$ & 0,451 \\
Escore Z IMC/I & $-0,5 \pm 1,2^{\mathrm{a}}$ & $0,2 \pm 1,0$ & 0,023 \\
Razão P/C & $62,8 \pm 7,7$ & $66,2 \pm 8,0$ & $0,2 \pm 1,1^{\mathrm{b}}$ & 0,075 \\
PC/idade & $0,06(0,05-0,07)$ & $0,06(0,06-0,07)$ & $0,07(0,06-0,07)$ & 0,094 \\
PC & $34,0(32,0-35,5)$ & $34,0(33,0-35,0)$ & $35,0(33,5-36,0)$ & 0,100 \\
PC/Idade & $-0,2 \pm 1,6$ & $-0,3 \pm 1,1$ & $0,2 \pm 1,3$ & 0,270 \\
\hline C & & &
\end{tabular}

$\mathrm{C} / \mathrm{I}=$ comprimento para idade; $\mathrm{CN}$ = comprimento ao nascer; $\mathrm{IMC}$ = índice de massa corpórea; IMC/I = índice de massa corpórea para idade; IP = índice ponderal; $\mathrm{PC}=$ perímetro cefálico; $\mathrm{P} / \mathrm{C}$ = peso para o comprimento; $\mathrm{PC} / \mathrm{ldade}=$ perímetro cefálico para idade; $\mathrm{P} / \mathrm{I}=$ peso para idade; $\mathrm{PN}=$ peso ao nascer; Razão $\mathrm{P} / \mathrm{C}=$ razão peso para o comprimento.

*Teste ANOVA One Way (comparação de médias), com teste de Tukey a posteriori; Teste Kruskal-Wallis (comparação de medianas).

cardíaco, entretanto, metade dos portadores de cardiopatias congênitas deve ser operada no primeiro ano de vida?

As crianças apresentaram condição nutricional ao nascimento relativamente preservada, com prevalências de distúrbios nutricionais semelhantes às descritas na literatura para crianças saudáveis. Foi evidenciado por outros autores que crianças que nascem com cardiopatia congênita, em sua maioria, não apresentam comprometimento do peso ao nascimento, sendo este indicador afetado posteriormente ${ }^{6,15}$.

Os resultados desse estudo revelam prevalência de baixo peso ao nascer $(6,8 \%)$ similar a encontrada em crianças no Nordeste brasileiro sem cardiopatia congênita $(7,4 \%)^{15}$, bem como de crianças com cardiopatia congênita $(8,6 \%)^{11}$. Provavelmente por ser realizado somente com crianças nascidas a termo, e as mães em sua maioria $(96,3 \%)$ terem realizado prénatal, condições relevantes para uma boa condição nutricional ao nascimento, colaboraram para esta baixa prevalência.

O peso ao nascer é o parâmetro nutricional mais utilizado para avaliar o recém-nascido, pois reflete as condições de saúde e nutrição do feto e da mãe, sendo um importante preditor de morbimortalidade e relaciona-se com o crescimento e desenvolvimento futuro da criança $^{16}$. Daí a importância de ser avaliado, assim como outras medidas antropométricas, pois permite a identificação do estado nutricional e sua associação com a morbi-mortalidade ${ }^{17}$.

A associação das medidas, gerando indicadores, é tão importante quanto as medidas sequenciadas. $O \mathrm{IP}$ é a estratégia de avaliação utilizada para relacionar as diferentes dimensões do crescimento fetal na desnutrição, principalmente no RCIU. Neste estudo, observou-se que $28,2 \%$ das crianças eram desproporcionais, portanto baixo peso e comprimento adequado, o que indica déficit de crescimento no final da gestação. Este resultado é relativamente similar aos dados encontrados por Lima et al. ${ }^{18}$ também com crianças nascidas a termo, mas sem cardiopatias $(24,5 \%)$. A desproporcionalidade é um fator comumente associado a baixo peso ou estatura ao nascimento.

O perímetro cefálico, por sua vez, reflete o tamanho do encéfalo e à medida que aumenta, proporcionalmente, indica um bom crescimento e bom prognóstico neurológico ${ }^{17}$. Essa investigação apontou 15,2\% de crianças com comprometimento dessa medida, percentual inferior aos dados descritos por Anderson e Cunha ${ }^{19}$, que por meio da realização da comparação de duas coortes revelaram que $21,6 \%$ e $24,6 \%$ das crianças nasceram com o PC abaixo da normalidade nos anos de 1978/1979 e 2010, respectivamente. As diferenças nestes resultados podem ser justificadas pela diminuição do ponto de corte para a normalidade do perímetro cefálico propostas pelo Ministério da Saúde (e adotadas neste estudo), devido à epidemia de Zika vírus ocorrida no Brasil nos últimos anos, em que houve um aumento expressivo dos casos de microcefalia no país. A medida do PC é um marcador importante de crescimento cerebral adequado, crianças com PC abaixo da média podem apresentar atraso no desenvolvimento neuropsicomotor e cognitivo ${ }^{20}$.

Nossos achados apontaram ausência de diferenças entre os parâmetros nutricionais segundo o sexo, ao contrário dos dados apresentados por Monteiro et al. ${ }^{21}$, que verificaram dimensões corporais menores no sexo feminino ao nascimento em relação ao sexo masculino, e Viana et al. ${ }^{22}$, que reportaram maiores prevalências de baixo peso ao nascer e peso insuficiente entre as meninas.

Menores valores de escore $\mathrm{Z}$ dos indicadores $\mathrm{P} / \mathrm{I}$ $(p=0,030)$ e IMC/I $(p=0,023)$ para as cardiopatias cianóticas, quando comparadas aos outros tipos de cardiopatia congênita foram observados em nosso estudo, e também 
é descrito por outros autores com crianças maiores, apontando que a cianose influencia negativamente a condição nutricional da criança, levando ao déficit pôndero-estatural ${ }^{23}$. Nossos resultados sugerem, portanto, que as cardiopatias que cursam com cianose podem influenciar negativamente o crescimento dos portadores da doença ainda na fase fetal. $\mathrm{O}$ indicador $\mathrm{P} / \mathrm{I}$ permite avaliar alterações de peso precocemente, sendo um parâmetro bem preciso até os dois anos, já que nessa fase as deficiências nutricionais afetam mais o peso do que a estatura, enquanto o indicador IMC/I reflete falha no desenvolvimento estatural e ponderal.

No Brasil, todos os recém-nascidos devem ser avaliados ainda na sala de parto, incluindo peso, comprimento e os perímetros cefálico, torácico e abdominal, dados que deverão constar na Caderneta de Saúde da Criança (CSC), fornecida nas maternidades pelo Ministério da Saúde, para o acompanhamento do crescimento e desenvolvimento da criança até os 10 anos de idade. Como não existe curva de crescimento específica para crianças com cardiopatia congênita, todos deverão ser acompanhados por esta ferramenta.

Atualmente, as cardiopatias congênitas tem grande impacto nas taxas de mortalidade perinatal e infantil no Brasi 19,10 , sendo, portanto, de extrema necessidade a melhoria da assistência a estas crianças em nosso país desde o nascimento, pois o período neonatal é momento de grande vulnerabilidade, havendo necessidade de cuidados especiais, com importância fundamental para a redução da mortalidade infantil. Para tanto, se faz necessária a criação de políticas públicas para otimizar o tratamento e diminuir a fila de espera para a cura da doença ${ }^{24}$. No Brasil, são necessários mais de onze mil novos procedimentos/ano, com um déficit estimado em $80 \%$, sendo maior nas regiões Norte e Nordeste do país?. Só no Estado de São Paulo, que possui 12 Serviços de cirurgia cardíaca pediátrica, o déficit é de 54\% em menores de um ano de idade ${ }^{10}$. Em 2009, no Estado de Pernambuco, que possui três centros para tratamento cirúrgico da cardiopatia congênita, o déficit foi estimado em $72,73 \%{ }^{24}$, diferentemente do que ocorre na Espanha, onde os defeitos são prontamente corrigidos, tão logo seja necessário ${ }^{25}$. A literatura ainda é escassa, quanto aos dados nutricionais de nascimento de crianças com cardiopatia congênita, sobretudo sobre a proporcionalidade corporal, o que dificultou a discussão dos achados, entretanto, poderá servir como base comparativa para futuras investigações.

\section{CONCLUSÃO}

Crianças com cardiopatias congênitas apresentaram condição nutricional ao nascimento relativamente preservada, com prevalência de distúrbios nutricionais semelhante às descritas na literatura. Entretanto, foram observadas menores médias de escore $Z$ dos indicadores $\mathrm{P} / \mathrm{I}$ e IMC/I para as cardiopatias cianóticas, quando comparadas aos outros tipos (acionáticas ou mistas), sugerindo, portanto, que as cardiopatias que cursam com cianose podem influenciar negativamente o crescimento dos portadores da doença ainda na fase fetal.

\section{REFERÊNCIAS}

1. Pinto Júnior VC, Branco KMPC, Cavalcante RC, Carvalho Junior W, Lima JRC, Freitas SM, et al. Epidemiology of congenital heart disease in Brazil. Braz J Cardiovasc Surg. 2015;30(2):219-24.

2. Amorim LFP, Pires CAB, Lana AMA, Campos AS, Aguiar RALP, Tibúrcio JD, et al. Apresentação das cardiopatias congênitas diagnosticadas ao nascimento: análise de 29.770 recémnascidos. J Pediatr (Rio J). 2008;84(1):83-90.

3. Miyague NI, Cardoso SM, Meyer F, Ultramari FT, Araújo FH, Rozkowisk I, et al. Estudo epidemiológico de cardiopatias congênitas na infância e adolescência: análise em 4538 casos. Arq Bras Cardiol. 2003;80(3):269-73.

4. Belo WA, Oselame GB, Neves EB. Perfil clínico-hospitalar de crianças com cardiopatia congênita. Cad Saúde Colet. 2016;24(2):216-20.

5. Leite HP, Carvalho ACC, Fisberg M. O estado nutricional de crianças portadoras de cardiopatia congênita com shunt esquerda-direita: importância da presença de hipertensão pulmonar. Arq Bras Cardiol. 1995;65(5):403-7.

6. Blasquez A, Clouzeau H, Fayon M, Mouton JB, Thambo JB, Enaud R, et al. Evaluation of nutritional status and support in children with congenital heart disease. Eur J Clin Nutr. 2016;70(4):528-31.

7. Miller HC, Hassanein K. Diagnosis of impaired fetal growth in newborn infants. Pediatrics. 1971;48(4):511-22.

8. Uchimura TT, Szarfarc SC, Uchimura NS, Bercini LO. Índice de proporcionalidade do baixo peso ao nascer e a sua relação com a mortalidade neonatal. Acta Scientiarium. 2001;23(3):753-7.

9. Pinto Júnior VC, Rodrigues LC, Muniz CR. Reflexões sobre a formulação de política de atenção cardiovascular pediátrica no Brasil. Rev Bras Cir Cardiovasc. 2010;24(1):73-80.

10. Caneo LF, Jatene MB, Yatsuda N, Gomes WJ. Uma reflexão sobre o desempenho da cirurgia cardíaca pediátrica no Estado de São Paulo. Rev Bras Cir Cardiovasc. 2012;27(3):457-62.

11. Kramer HH, Trampisch HJ, Rammons S, Giese A. Birth weight of children with congenital heart disease. Eur J Pediatr. 1990;149(11):752-7.

12. WHO Multicentre Growth Reference Study Group. WHO child growth standards: methods and development: length/height-forage, weight-for-age, weight-for-length, weight-for-height and body mass index-for-age. Geneva: World Health Organization; 2006. [cited 2019 Nov 12]. Available from: https://www.who. int/childgrowth/standards/technical_report/en/

13. Leão Filho JC, Lira PIC. Estudo da proporcionalidade corporal de recém-nascidos a termo segundo o índice ponderal de Rohrer e grau de retardo de crescimento intra-uterino. Cad Saúde Pública. 2003;19(6):1603-10.

14. World Health Organization. Construction of the head circumference-for-age standards. WHO child growth standards: head circumference-for-age, arm circumference-for-age, triceps skinfold-for-age and subscapular skinfold-for-age: methods and development. Geneva: World Health Organization; 2007. [cited 2019 Nov 12]. Available from: https://www.who.int/ publications-detail/9789241547185

15. Pedraza DF. Baixo peso ao nascer no Brasil: revisão sistemática de estudos baseados no sistema de informações sobre nascidos vivos. Revista de Atenção à Saúde. 2014;12(41):37-50. 
16. Tourinho AB, Reis LBSM. Peso ao nascer: uma abordagem nutricional. Com Ciências Saúde. 2013;22(4):19-30.

17. Falcão MC. Avaliação nutricional do recém-nascido. Pediatria. 2000;(22):235-9.

18. Lima MC, Dantas HF, Amorim RJM, Lira PIC. A restrição do crescimento fetal influência a composição corporal na idade escolar? J Pediatr. 2011;87(1):29-35.

19. Anderson D, Cunha V. Distribuição das medidas de perímetro cefálico das coortes de nascidos em Ribeirão Preto em 1978/79 e em 2010. São Paulo, 2013. [cited 2019 Nov 12]. Available from: file://C:/Users/claudia.sabino/Downloads/Daniela\%20 e\%20Val\%C3\%A9ria.pdf

20. Brasil. Ministério da Saúde. Secretaria deAtenção à Saúde. Protocolo de atenção à saúde e resposta à ocorrência de microcefalia relacionada à infecção pelo vírus Zika. Brasília: Ministério da Saúde; 2015. p.49. [cited 2019 Nov 12]. Available from: https:// www.saude.gov.br/images/PROTOCOLO\%20DE\%20ATENDIMENTO\%20PARA\%20MICROCEFALIA.pdf
21. Monteiro FPM, Araújo TL, Lopes MVO, Chaves DBR, Beltrão BA, Costa GSA. Estado nutricional de crianças com cardiopatias congênitas. Rev Latino-Am Enfermagem. 2012;20(6):1024-32.

22. Viana KJ, Taddei JAAC, Cocetti M, Warkentin S. Peso ao nascer de crianças brasileiras menores de dois anos. Cad Saúde Pública. 2013;29(2):349-56.

23. Pinheiro DGM, Pinheiro CHJ, Marinho MJF. Comprometimento do desenvolvimento pondo-estatural em crianças portadoras de cardiopatias congênitas com shunt cianogênico. Rev Bras Promoç Saude. 2008;21(2):98-102.

24. Pinto Júnior VC, Fraga MNO, Freitas SM, Croti UA. Regionalização da cirurgia cardiovascular pediátrica brasileira. Rev Bras Cir Cardiovasc. 2013;28(2):256-62.

25. Jordan BH. Control de los niños con cardiopatía congénita en atención primaria. Rev Pediatr Aten Primaria. 2009;11(4):639-55.

Local de realização do estudo: Pronto Socorro Cardiológico Universitário de Pernambuco, Universidade de Pernambuco, Recife, PE, Brasil.

Conflito de interesse: Os autores declaram não haver. 